\title{
GPPS-BJ-2019-0013
}

\section{Variable Blade Tip Geometry Inserts for Combined Aerothermal Measurements for Bladed Disk Rotors}

\author{
Dominic D. Hänni \\ Laboratory for Energy Conversion, ETH Zurich \\ dohaenni@ethz.ch \\ Zurich, Switzerland \\ Reza S. Abhari \\ Laboratory for Energy Conversion, ETH Zurich \\ rabhari@lec.mavt.ethz.ch \\ Zurich, Switzerland
}

\author{
Rainer Schädler \\ Laboratory for Energy Conversion, ETH Zurich \\ schaedler@lec.mavt.ethz.ch \\ Zurich, Switzerland
}

\author{
Anestis I. Kalfas \\ Aristotle University of Thessaloniki \\ akalfas@auth.gr \\ Thessaloniki, Greece
}

\section{ABSTRACT}

Cost effective experimental testing in turbomachinery requires the integration of multiple geometries in one rotor. So-called rainbow designs, combined with fast response aerodynamic and entropy probes, allow for experimentally investigating different designs in a timely and cost effective manner. The investigation of new blade tip geometries is of particular interest, because tip leakage losses account for approximately one third of the total aerodynamic losses in a turbine stage.

This work presents the design, integration, and testing of a variable and exchangeable blade tip setup integrated in a bladed disk rotor. The key features of the concept are high flexibility of the geometry, coupled with the possibility of introducing blade tip coolant flows in bladed disk arrangements, and the integration of tip heat transfer measurements. After successful bench tests, an existing rotor has been modified with three tip inserts of the same geometry, and subsequently tested in the axial turbine research facility LISA at ETH Zurich.

A custom-made thin film heater for optical highresolution heat transfer measurements was successfully integrated on the insert and tested during operation. Flow field measurements behind the rotor indicated that the aerodynamics of the rotor is not influenced by installing tip inserts. The setup has proven its robustness in more than 80 days of experimental testing, and has provided some encouraging tip heat transfer data.

\section{INTRODUCTION}

The aerodynamic efficiency and thermal life-time of gas turbines are still the main drivers for research and developments in turbomachinery. Rotor blade tips are of special interest, as tip leakage losses account for approximately one third of the total losses (Denton, 1993) and cooling them is difficult. Computational fluid dynamics (CFD), together with fully automatic parametric optimisations, are currently used to support the development of new designs. However, as discussed by (Denton, 2010), CFD has some limitations, meaning experimental testing is still required for validation.

Rainbow testing, using multiple designs on one rotor, is a cost and time efficient approach. (Rezasoltani et al., 2015) measured film cooling effectiveness using a rainbow setup for four tip geometries using pressure sensitive paint. They reported a strong interaction of the cooling jets with the leakage flows, where the characteristics differed between the flat and squealer tip. A rainbow setup for investigating tip geometry and tip gap influence was presented by (Lavagnoli et al., 2016), which underlined the importance of tip gap size for engine representative flows. With this setup, they achieved blade-to-blade variations in the tip radius below $\pm 0.1 \mathrm{~mm}$. The same setup was used by (Cernat et al., 2018) for the combined aerothermal testing of tip designs, where high-frequency pressure and temperature instrumentation at the rotor casing was used. The time average pressure and heat transfer at the casing was found to vary significantly with blade tip geometry, as a function of blade passage and tip gap position. However, heat transfer was found to be less affected by the tip geometry.

Most experimental tip geometry investigations are performed in linear cascades, because testing in rotating facilities is expensive and challenging. The work from (Nasir et al., 2004) showed that a blade tip squealer significantly alters the flow field at the tip compared to a flat baseline, and produces overall lower heat transfer coefficients. Even partial 
squealers (representing a degraded squealer in real engines) can be beneficial in terms of overall heat transfer coefficients. This has been confirmed by CFD investigations. For example, (Krishnababu et al., 2008) concluded that a cavity tip (squealer) is advantageous from both aerodynamic and heat transfer perspectives. Further, stage efficiency improvements of $0.9 \%$ were reported by (Zhou and Zhong, 2017) for simulations of an optimised suction side squealer geometry, when compared to a base line squealer.

Cascade experiments using trip stripes on a flat tip demonstrated a $10 \%-15 \%$ reduction of heat transfer coefficient, if the stripes were placed orthogonal to the leakage flow (Saxena et al., 2004). Cylindrical pin fins and strips along the leakage direction were even found to increase heat transfer coefficients by up to $20 \%$ in some cases. The potential of new tip geometries was also shown by (De Maesschalck et al., 2016) with extensive CFD simulations.

This work presents the design and testing of an insert based concept to perform combined aerothermal rainbow blade tip geometry studies with bladed rotor disks in a rotating facility. The setup is validated with aerodynamic probe measurements, and preliminary measurements of blade tip heat transfer measurements in the rotating frame of reference with an infrared based setup have been completed.

\section{VARIABLE BLADE TIP GEOMETRY INSERTS}

The use of bladed rotor disks (blisks) in experimental research facilities has many advantages. The single-part manufacturing allows tighter tolerances and leads to much faster assembly times for testing. Further, having no gaps between parts has a positive influence on high-resolution aerodynamic measurements. On the downside, geometry variations on existing blisks are not possible and are inherent with the complete manufacturing of new blisks, and are therefore expensive. Moreover, tip cooling integration (due to internal flow paths) would require a 3D printed blisk.

The intention of this work is to evaluate and build a robust, simple, and versatile design concept to modify existing blisk rotors for variable blade tip geometry investigations (including tip coolant ejection). Further, the integration of a previously developed high-resolution optical heat transfer measurement setup (using controlled thermal boundary conditions) should be possible, including temperature and pressure sensors in the tip.

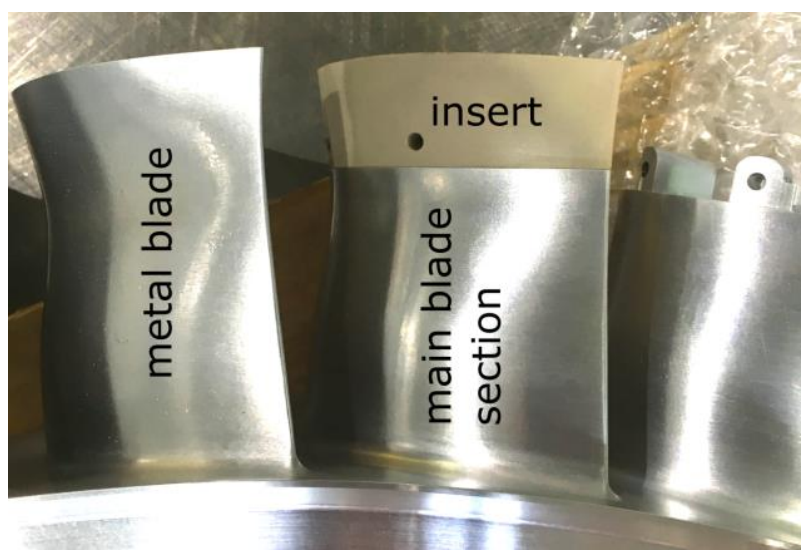

Figure 1 Rotor blisk with metal blade and split main blade with installed tip insert.

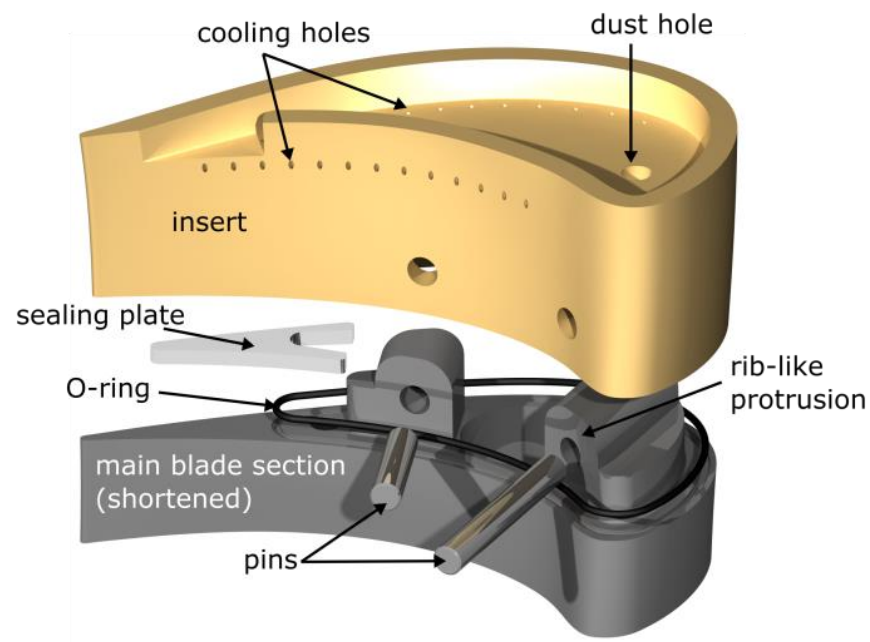

Figure 2 Schematic illustration of blade tip insert fixation on rotor blade main blade.

\section{Insert Design and Assembly}

Insert based modification and instrumentation was developed according to the design specifications. The blisk blade was split into two parts: the main blade section and tip insert (Figure 1), whereof the main blade section covers approximately $70 \%$ of the span height. This approach is convenient for simple geometry variations and instrumentation. The tip insert can be instrumented and calibrated separately of the whole blisk. It also allows the modification of existing blisks to integrate such inserts. Further, the integration of an internal cooling air supply hole from the disk cavity can be machined using conventional methods.

The tip insert was manufactured from polyether ether ketone (PEEK), which is a lightweight but high strength thermoplastic polymer (yield stress $\sim 110 \mathrm{MPa}$ ) with good machinability. The low thermal conductivity and electrical conductance are beneficial for the heat transfer measurement setup. To allow for blade tip coolant ejection, the insert is hollow and provides a plenum to provide coolant to the cooling and dust holes.

The assembly and fixation of the insert on the main blade section is schematically depicted in Figure 2. Two pins are used to fix the insert to the main blade section (approximately at $25 \%$ and $55 \%$ of the camber line starting from the leading edge). For this purpose, the main blade section has two riblike protrusions on the joint face to hold the pins and the centrifugal load of the insert. The shape of the protrusions, and the tight fit to the insert, ensure precise alignment. The angles of the fixation pins maximise the contact area with the insert shell, while also ensure accessibility for the installation and removal of the pins considering the neighboring blades. Epoxy glue was used to secure the pins in the insert shell, and to smooth the flow exposed surface of the pin-hole. The borehole into the disk cavity (for the cooling supply) is located between the two protrusions. This is not visible in the figure. The channel for instrumentation cables was placed behind the trailing edge protrusion, and the hollow insert in the trailing edge area was closed with a little sealing plate. A $0.7 \mathrm{~mm} \mathrm{O-}$ ring was installed between the main blade section and the insert on the joint face to minimise coolant leakages. 


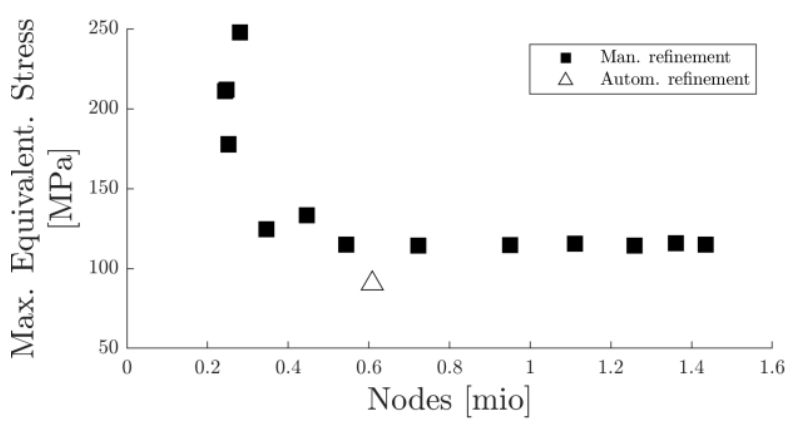

\section{Figure 3 FEM mesh sensitivity of maximum von Mises stress in the fixation pin.}

\section{Mechanical Integrity of Insert Fixation}

Mechanical integrity of the insert is crucial for safe operation inside a rotating facility. Forces due to rotation and plenum overpressure, in addition to bending forces due to pressure differences, are the main loads to be absorbed by the insert and the fixation. The latter is carried successfully by the protrusions of the main blade section.

Preliminary FEM simulations with Ansys 18.2 were performed to determine the effect of radial loading on the insert, and its displacement from the main blade section. The assembly depicted in Figure 2, excluding the trailing edge sealing plate and O-ring, was used and modelled with a fixed support at the blade main section bottom and a rotational velocity. The simulations were performed with an estimated insert weight (including instrumentation) of $13 \mathrm{~g}$ (subsequently measured as $13.22 \mathrm{~g}$ ), and a turbine design speed of $2700 \mathrm{rpm}$. The calculated centrifugal load on the fixation was $400 \mathrm{~N}$ for these conditions. A mesh sensitivity study (Figure 3) using the maximum von Mises stresses and deformations was performed comparing automatic mesh refinement to manual overall mesh settings. Based on this, the model with the automatic refined mesh of 0.6 million nodes was used for the simulations. The maximum calculated equivalent stress are below the material limits (e.g. $33 \mathrm{MPa}$ is $30 \%$ of the material yield strength for the insert). Therefore, the FEM simulations were predicting the mechanical integrity of the setup during operation.
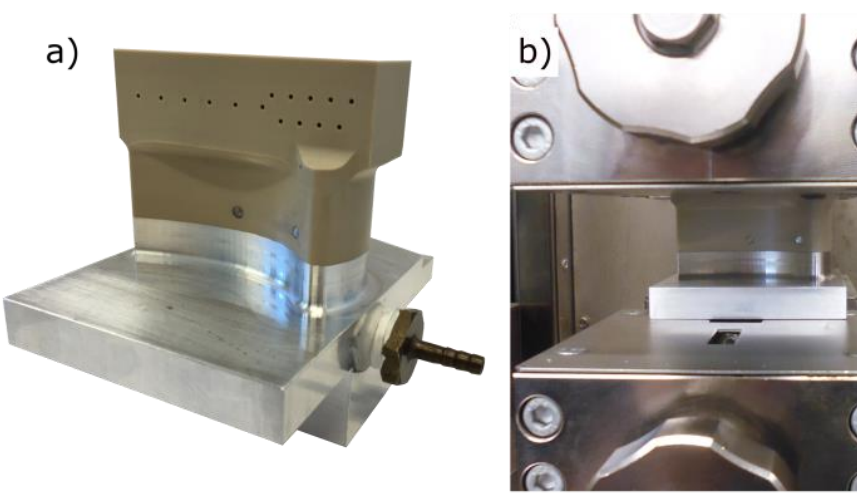

To validate the predictions from the simulations experimentally, a test assembly (Figure 4) was built and tested in a tensile testing machine. This assembly represented the setup in terms of blade geometry, insert fixation, and materials. Ribs for clamping in the test machine were added at the blade section and insert. Static tensile tests up to $1200 \mathrm{~N}$ (SF 2.4) were performed for two different load ramping rates ( 2 and $5 \mathrm{~N} / \mathrm{s}$ ), and at an elevated temperature of $55^{\circ} \mathrm{C}$, which was representative for the test facility. All tests confirmed the mechanical integrity of the insert and fixation.

To assess the potential of rubbing at the casing, the total deformation of the insert with the given fixation was investigated. The nominal tip gap of the turbine is $0.7 \mathrm{~mm}$ (with a $\pm 50 \mu \mathrm{m}$ manufacturing tolerance) for the metal blades. In Figure 5, the total deformation for design rotational speed is depicted (scale factor 40). The insert was securely held in place around the two fixation locations. As expected, due to the limited fixation, the trailing edge parts exhibited the highest deflection: $21 \%$ of the nominal tip gap. Considering over-speed conditions during start-up (3000 rpm), the maximum deformation increased to $25 \%$ of the nominal tip gap. The effect on the flow field of this reduction in tip gap towards the trailing edge could not be predicted a priori. However, deformations between different designs for rainbow experiments are expected to be comparable.

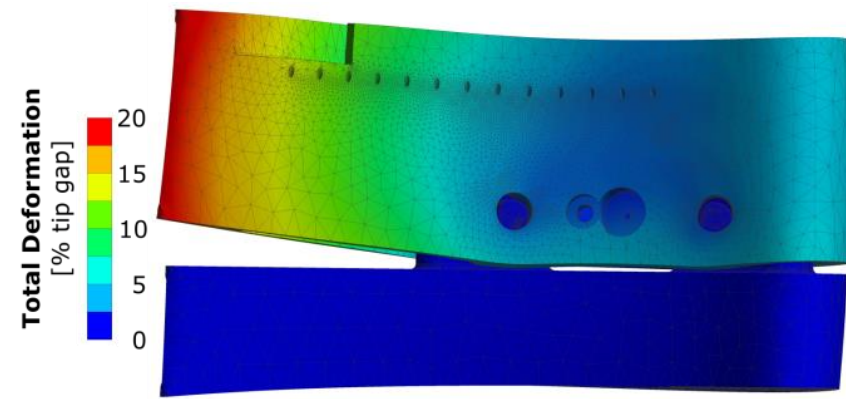

Figure 5 Predicted insert deformation under design operating conditions (scale factor 40 ).

\section{Parasitic Leakage Losses}

The variable blade tip inserts were also used for studies on tip coolant ejection. Therefore, the assembly should be free of parasitic leakage between the insert and the main blade section. Preliminary leakage tests with the test assembly showed that relevant leakage through the joint surface occurred without the installation of additional sealing. Tests were conducted in the tensile stress machine with an installed sealing O-ring, an insert plenum overpressure, and a representative load of $500 \mathrm{~N}$. The measured leakage rates, as a function of overpressure, are presented in Figure 6. The maximum blade specific leakage rate of $1.2 \mathrm{e}-4 \mathrm{~g} / \mathrm{s}$ (for 950 mbar overpressure) was four orders of magnitude lower than the estimated tip coolant ejection rates of $1.2 \mathrm{~g} / \mathrm{s}$ per blade after the first experiments. Therefore, the assembly was considered sufficiently sealed against parasitic leakage.
Figure 4 Test assembly (a) for preliminary test mounted in tensile test machine (b). 


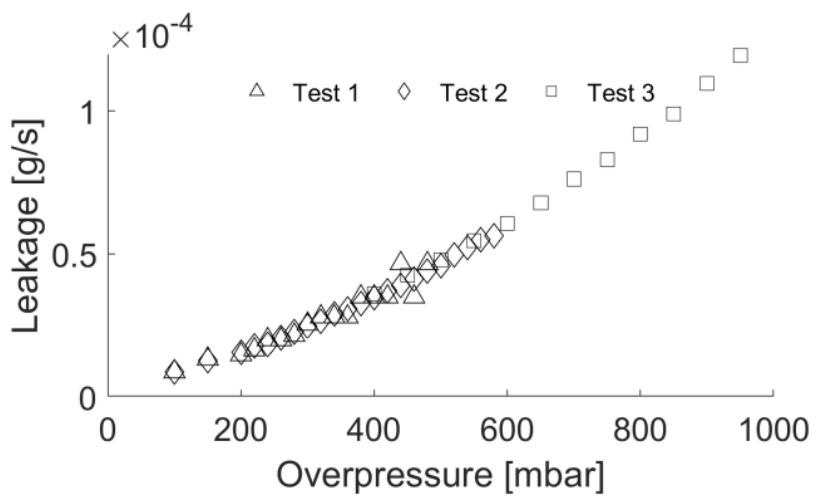

Figure 6: Measured leakage rate $[\mathrm{g} / \mathrm{s}]$ as function of overpressure and $500 \mathrm{~N}$ tensile load.

\section{EXPERIMENTAL SETUP AND METHODOLOGY}

\section{Research Facility}

The instrumentation presented in this paper was designed for (and tested in) the axial turbine research facility "LISA" at ETH Zurich. The test rig is a continuously running, quasiclosed loop rotating test rig with non-dimensional parameter matching real-engine conditions (Figure 7). A radial compressor drives the air of the low-temperature and moderate-speed facility. A two-stage water-to-air heat exchanger after the compressor controls the turbine total inlet temperature to within an accuracy of $\pm 0.4 \mathrm{~K}$. Before the test section, a flow conditioning stretch ensures uniformity of the inlet flow. After the turbine section, two rows of de-swirling vanes recover the static pressure, as the loop is open to atmosphere, before the flow is fed back to the compressor through a calibrated venturi nozzle for mass flow measurements. The rotational speed is controlled with a DC generator, connected through a reduction gearbox with the turbine shaft, with an accuracy of $\pm 0.02 \%$ ( $\pm 0.5 \mathrm{rpm})$. A more detailed description of the test facility is given by (Sell et al., 2001) and (Behr et al., 2006).

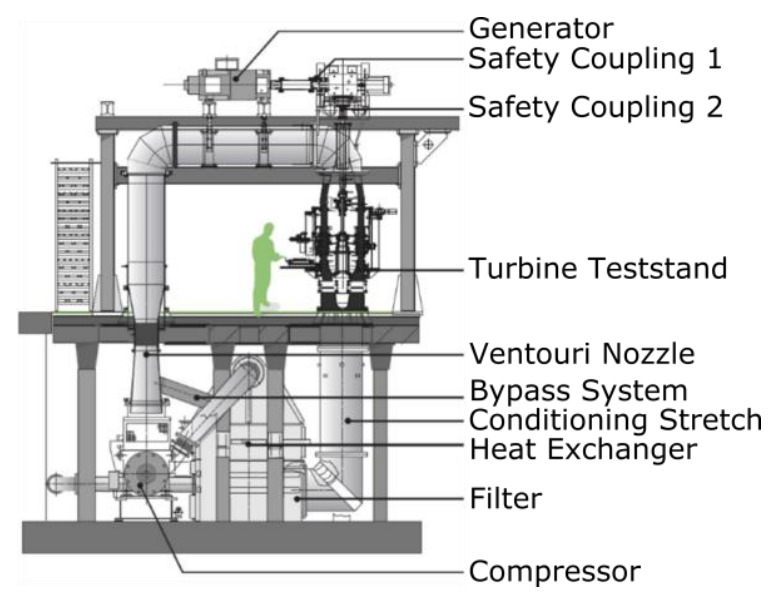

Figure 7 Overview of the axial turbine research facility "LISA" at ETH Zurich. a)

squealer cooling holes
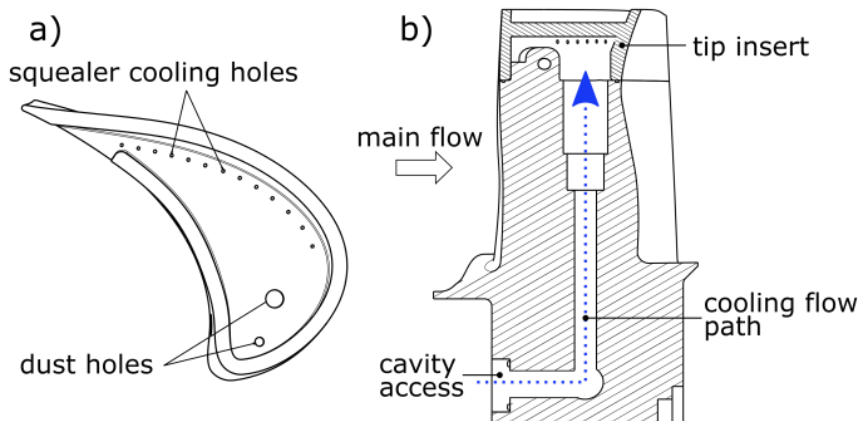

Figure 8 Tip cooling hole arrangement (a) and coolant supply path (b).

\section{Turbine Configuration and Operating Conditions}

The test case used for testing the novel rotor blade tip inserts is a 1.5-stage high-pressure turbine representative design with 3D optimised geometries and endwall contouring. The rotor squealer cavity depth is $5 \%$ of the blade span, and the fence has a uniform thickness of 3.5\%. More details about the design is given by (Schädler et al., 2019). In total, 26 cooling holes with a diameter of $0.5 \mathrm{~mm}$ are distributed over the tip. Half of these are at the blade pressure side, and half are in the squealer recess towards the suction side fence. Two dust holes of 0.75 and $1.5 \mathrm{~mm}$ in diameter are placed in the squealer recess towards the leading edge (Figure 8 ).

The turbine was operated with a constant total to static pressure ratio accounting for changes in atmospheric pressure. The pressure ratio was $\Pi_{1.5}=1.65$ for the aerodynamic probe measurements, and $\Pi_{1.5}=1.33$ for the heat transfer measurements, with a constant rotational speed of $2700 \mathrm{rpm}$. More details on the two operating conditions are summarized in Table 1.

Measurements were performed both with and without tip coolant ejection. The coolant was supplied from the rotor upstream cavity with two bore holes into the tip insert plenum, schematically illustrated in Figure 8. For the measurements without active tip coolant supply, the cavity access was sealed with a plate. The measurement conditions are labelled TR1 for tip coolant, and TR0 without coolant ejection.

Table 1: Rig operating conditions

\begin{tabular}{|c|c|c|c|}
\hline Parameter & OP1 & OP2 & Units \\
\hline$\Pi_{1.5}$ & $1.65 \pm 0.4 \%$ & $1.33 \pm 0.4 \%$ & {$[-]$} \\
\hline$T_{t, i n}$ & \multicolumn{2}{|c|}{$328 \pm 0.3$} & {$[\mathrm{~K}]$} \\
\hline Mass flow & $151.8 \pm 0.2 \%$ & $123.6 \pm 0.2 \%$ & {$\left[\frac{k g \sqrt{K}}{c h r}\right]$} \\
\hline Rotor speed & \multicolumn{2}{|c|}{$2.48 \pm 0.05$} & {$\left[\frac{r p s}{\sqrt{K}}\right]$} \\
\hline $\begin{array}{l}\text { Mach number } \\
\left(\mathrm{S} 1_{\mathrm{ex}} / \mathrm{R}_{\mathrm{ex}} / \mathrm{S} 2_{\mathrm{ex}}\right)\end{array}$ & $0.53 / 0.27 / 0.48$ & $0.43 / 0.16 / 0.34$ & {$[-]$} \\
\hline $\operatorname{Re}(\mathrm{S} 1 / \mathrm{R} / \mathrm{S} 2)$ & $7.1 / 3.8 / 5.1 \cdot 10^{5}$ & $5.2 / 3.1 / 4.6 \cdot 10^{5}$ & {$[-]$} \\
\hline
\end{tabular}

\section{Fast Response Aerodynamic Probe (FRAP)}

Aerodynamic flow field measurements behind the instrumented rotor blades were performed with a fast-response aerodynamic probe (FRAP). The probe technology has been developed by the laboratory of energy conversion (LEC) at ETH, and is commercialised by Limmat Scientific AG. The 
measurement technology is described in more detail in (Kupferschmied et al., 2000) and (Pfau et al., 2003).

The two sensor miniature probes $(1.8 \mathrm{~mm}$ tip diameter $)$ were operated in a virtual four sensor mode, as described by (Pfau et al., 2002), to derive the flow velocities and angles. The unsteady pressure signal was acquired with a sampling rate of $200 \mathrm{kHz}$. With the simultaneously recorded shaft trigger signal, specific blade passing events can be reconstructed in the post-processing; hence, the data can be compared between instrumented and metal blades. The measurement grid contained 37 points in a radial direction (clustered towards the hub and tip endwall) and 41 points in a circumferential direction (covering one stator pitch of $10^{\circ}$ ). The distance between the rotor trailing edge and the probe was $10.4 \%$ of the axial chord length of the rotor.

\section{Heat Transfer Measurement Setup}

An infrared based measurement technique was used to perform heat transfer measurements on the rotor blade tip. The publications from (Lazzi Gazzini et al., 2017b), (Lazzi Gazzini et al., 2017a) and (Hänni et al., 2019) provide a detailed description of the setup, which was adopted for the blade tip measurements and is only briefly outlined here.

A high-speed infrared camera (FLIR SC7300L) recorded the surface temperature of the blade tip through an infrared transparent window (Zinc-Selenide, $\mathrm{ZnSe}$ ) in the rotor casing. Camera recordings were triggered with a turbine shaft trigger, and consisted of 1000 triggered images per sequence. In the post-processing, only images of each sequence within a \pm 1 pixel jitter were averaged and considered for the further analysis.

High resolution surface temperature measurements $\left(T_{w}\right)$ were performed for multiple (typically 5 to 7) known quasi iso-energetic boundary conditions. The linear relation of the convective heat flux to the driving temperature difference is used to determine the heat transfer coefficient and adiabatic wall temperature (Equation 1). Using all measurement steps, a linear square fitting of the data according to Equation 1 is performed, illustrated in Figure 9.

$$
\dot{q}_{\text {conv }}^{\prime \prime}=h \cdot\left(T_{w}-T_{a w}\right)
$$

The convective heat flux is calculated from the known generated joule heat flux subtracting the radiative and conductive (measured with embedded temperature sensors) heat flux.

For the controlled boundary conditions, a thin film heater was integrated into the blade tip insert. Accordingly, a thin Nickel film was chemically deposited on the PEEK, and a heating track was cut using pulsed laser ablation (Figure 9a). For increased infrared signature and reduced reflections, the tip insert (as well as the whole rotor for comparable roughness) were painted with a high emissivity coating, and reflective infrared markers were positioned for image reconstruction (Figure 9b).

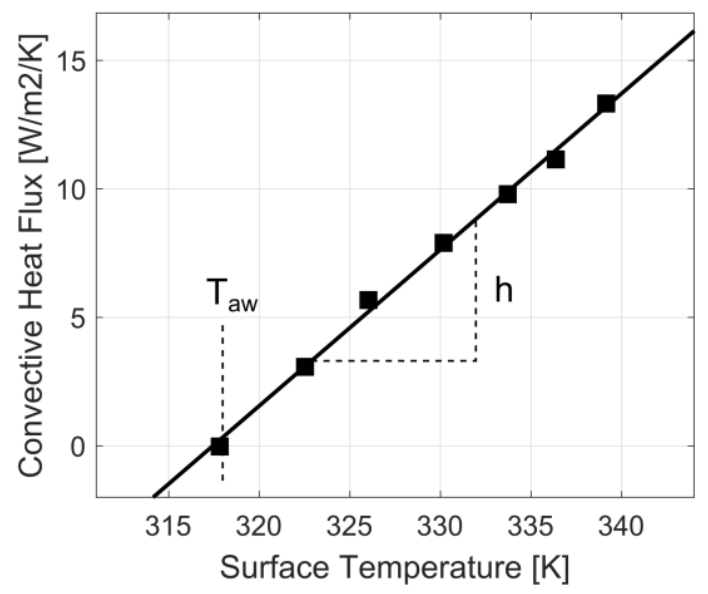
Figure 9 Illustration linear fit to derive heat transfer
coefficient $\mathrm{h}$ from measurements.

a)

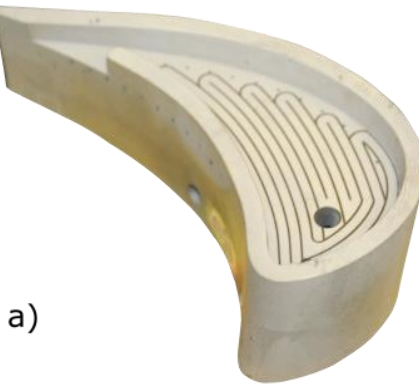

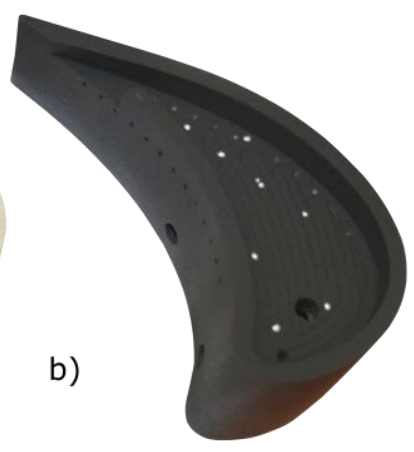

Figure 10 Thin film coated tip insert with heating track (a) and painted ready for installation (b).

\section{RESULTS AND DISCUSSION}

The present work focuses on the application of variable blade tip inserts on the rotor blisk for aerothermal measurements in rotating facilities. In the first section, results from the aerodynamic probe measurements comparing the flow field downstream instrumented blades and original metal blades are presented. In the second section, the initial preliminary results from the high-resolution tip heat transfer measurements on the inserts are presented.

\section{Insert Influence on Rotor Exit Flow Field}

To evaluate the differences between metal and insert instrumented blades, the time resolved data was split and analysed for each blade passing separately. Figure 10 shows the mass- and circumferentially-averaged relative total pressure measured at the rotor exit. The figure shows the averaged data of three instrumented blades against the average of all metal blades, together with the maximum and minimum values of blade-to-blade variations. As shown, the differences of the mean relative total pressure were small between the metal blades and instrumented blades. Considering the 


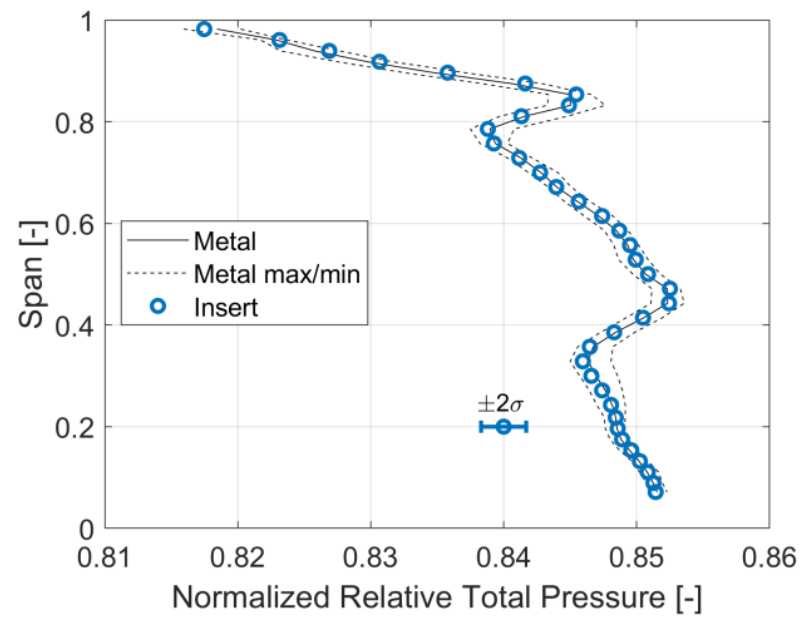

\section{Figure 11 Circumferentially mass averaged relative total pressure at rotor exit.}

measurement uncertainty ( $\pm 2 \sigma$ indicated) and the blade to blade variations, the influence of the tip inserts is concluded to be minimal.

Similar results were also found for the relative yaw angle at the rotor exit, as depicted in Figure 11. The angles for the instrumented blades closely followed the averaged values of the metal blades. The highest deviations were found in the tip region between $70 \%$ to $100 \%$ span, with up to $0.5^{\circ}$ difference. However, this is again below the blade-to-blade variation and the expanded measurement uncertainty

\section{Blade Tip Heat Transfer Measurements}

Preliminary results from the optical heat transfer measurements on the rotor blade tip are presented below for the described operating points. The data is shown without absolute scale (but scaling is consistent within the figures), as the focus of this research paper is on the instrumentation method.

Distributions of local heat transfer coefficients, both with and without active coolant ejection, are depicted in Figure 12. An influence of tip coolant flow on local heat transfer rate was captured and clearly visible in the data. In presence of tip coolant (TR1), a band of enhanced heat transfer rate was

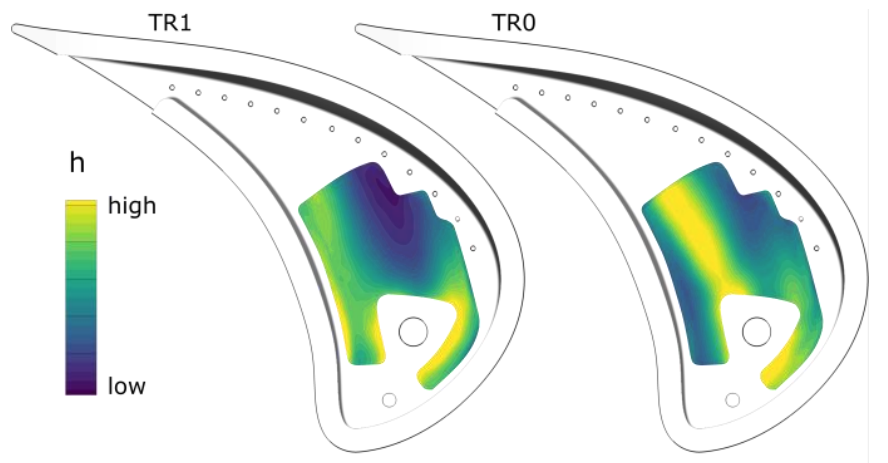

Figure 13 Heat transfer coefficient distribution for TR1 (a) and TR0 (b).

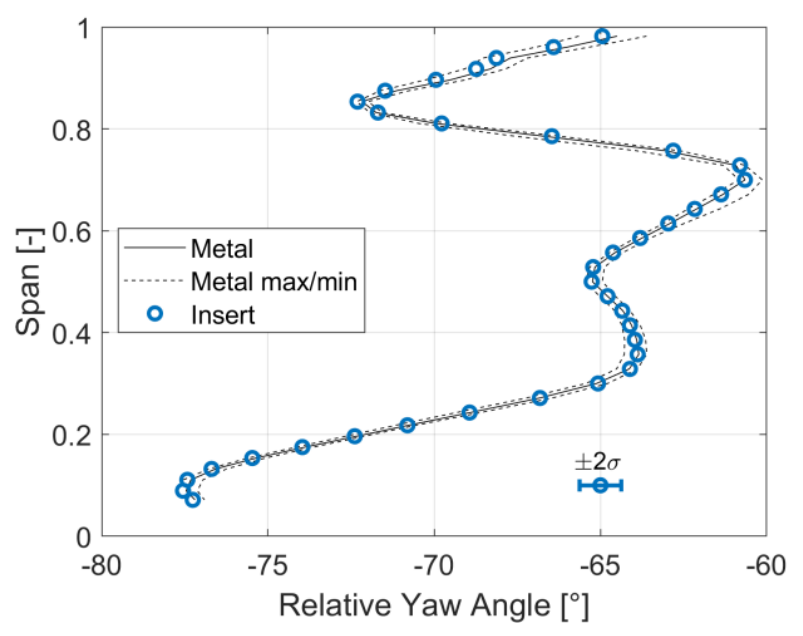

\section{Figure 12 Circumferentially mass averaged relative yaw angle at rotor exit.}

observed on the pressure side immediately behind the squealer fence. For the case without tip coolant ejection (TR0), this band shifted away from the pressure side towards the middle of the squealer recess area. The authors speculate that this could be caused by the recirculation vortex formed by the leakage flowing over the squealer fence, where the presence of coolant in the recess area shifts the vortex towards the pressure side.

Downstream from the big purge hole, an extended region of low heat transfer was found for tip coolant ejection. This low heat transfer region was not particularly clearly present in the case without coolant ejection (TR0).

Due to the overall small temperature drop over the rotor, differences in local adiabatic wall temperatures were also very small (below $3 \mathrm{~K}$ ). However, differences between the cases of with tip coolant ejection and without tip coolant are clearly visible, as shown in Figure 13 (same temperature scale). The ejection of coolant from the two dust holes resulted in lower adiabatic wall temperatures in the surroundings. Overall higher temperatures and a general temperature gradient in the axial direction were observed without coolant.

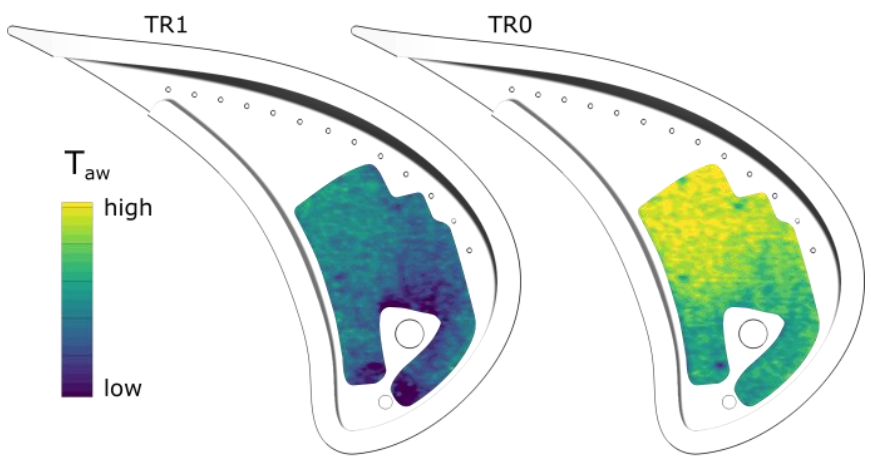

Figure 14 Adiabatic wall temperature distributions for TR1 (a) and TR0 (b). 


\section{CONCLUSIONS}

In this work, the design and testing of an instrumentation method to test variable blade tip geometries on bladed rotor disk was introduced. The presented blade tip inserts allow testing of variable blade tip geometries, in addition to tip coolant ejection, on a single blisk rotor. The setup was successfully tested in a rotating axial turbine test facility for more than 500 operating hours and more than 80 start-ups.

Time resolved flow field measurements did not reveal any significant changes in the flow field behind the inserts compared to metal reference blades, which underlines the viability of the concept for tip variation studies.

The inserts were successfully instrumented with a thin film surface heater and embedded temperature sensors to perform optical heat transfer measurements in the rotating frame of reference. Preliminary results show promising sensitivity of the setup to measure (and capture) local differences in heat transfer rate, and adiabatic wall temperature, in the case of variable blade tip coolant ejection.

The presented setup presents a promising approach for cost- and time-effective combined aerothermal investigations of blade tip geometries on bladed disk rotors.

\section{NOMENCLATURE}

\section{Variables}

$\begin{array}{lll}\mathrm{h} & \text { heat transfer coefficient } & {\left[\mathrm{W} / \mathrm{m}^{2} / \mathrm{K}\right]} \\ \Pi & \text { pressure ratio } & {[-]} \\ \dot{q}^{\prime \prime} & \text { convective heat flux } & {\left[\mathrm{W} / \mathrm{m}^{2}\right]} \\ \sigma & \text { standard error } & {[-] /\left[^{\circ}\right]} \\ \mathrm{T} & \text { temperature } & {[\mathrm{K}]}\end{array}$

\section{Subscripts}

aw adiabatic wall

conv convective

w wall

\begin{tabular}{ll}
\multicolumn{2}{l}{ Abbreviations } \\
FRAP & Fast response aerodynamic probe \\
LEC & Laboratory for Energy Conversion \\
SF & Safety factor \\
TR & Tip coolant ejection rate
\end{tabular}

\section{ACKNOWLEDGMENTS}

The authors would like to acknowledge the support of their industrial partners MTU Aero Engines AG and Siemens AG.

\section{REFERENCES}

BEHR, T., KALFAS, A. I. \& ABHARI, R. S. 2006. Unsteady Flow Physics and Performance of a One-and-1/2-Stage Unshrouded High Work Turbine. Journal of Turbomachinery, 129, 348-359.

CERNAT, B. C., PÁTÝ, M., DE MAESSCHALCK, C. \& LAVAGNOLI, S. 2018. Experimental and Numerical Investigation of Optimized Blade Tip Shapes-Part I: Turbine Rainbow Rotor Testing and Numerical Methods. Journal of Turbomachinery, 141, 011006-011006-13.
DE MAESSCHALCK, C., LAVAGNOLI, S., PANIAGUA, G., VERSTRAETE, T., OLIVE, R. \& PICOT, P. 2016. Heterogeneous Optimization Strategies for Carved and Squealer-Like Turbine Blade Tips. Journal of Turbomachinery, 138, 121011-121011-12.

DENTON, J. D. 1993. The 1993 IGTI Scholar Lecture: Loss Mechanisms in Turbomachines. Journal of Turbomachinery, 115, 621-656.

DENTON, J. D. 2010. Some Limitations of Turbomachinery CFD. 735-745.

HÄNNI, D. D., SCHÄDLER, R., ABHARI, R. S., KALFAS, A. I., SCHMID, G., LUTUM, E. \& N., L. 2019. Purge Flow Effects on Rotor Hub Endwall Heat Transfer with Extended Endwall Contouring into the Disk Cavity. Journal of the Global Power and Propulsion Society, 3, 1-13.

KRISHNABABU, S. K., NEWTON, P. J., DAWES, W. N., LOCK, G. D., HODSON, H. P., HANNIS, J. \& WHITNEY, C. 2008. Aerothermal Investigations of Tip Leakage Flow in Axial Flow Turbines-Part I: Effect of Tip Geometry and Tip Clearance Gap. Journal of Turbomachinery, 131, 011006011006-14.

KUPFERSCHMIED, P., KÖPPEL, P., GIZZI, W., RODUNER, C. \& GYARMATHY, G. 2000. Time-resolved flow measurements with fast-response aerodynamic probes in turbomachines. Measurement Science and Technology, 11, 1036.

LAVAGNOLI, S., DE MAESSCHALCK, C. \& ANDREOLI, V. 2016. Design Considerations for Tip Clearance Control and Measurement on a Turbine Rainbow Rotor With Multiple Blade Tip Geometries. V006T05A011.

LAZZI GAZZINI, S., SCHÄDLER, R., KALFAS, A. I. \& ABHARI, R. S. 2017a. Infrared thermography with nonuniform heat flux boundary conditions on the rotor endwall of an axial turbine. Measurement Science and Technology, 28, 025901-025901-15.

LAZZI GAZZINI, S., SCHÄDLER, R., KALFAS, A. I., ABHARI, R. S., HOHENSTEIN, S., SCHMID, G. \& LUTUM, E. 2017b. Effect of purge air on rotor endwall heat transfer of an axial turbine. Journal of the Global Power and Propulsion Society, 1, 211-223.

NASIR, H., EKKAD, S. V., KONTROVITZ, D. M., BUNKER, R. S. \& PRAKASH, C. 2004. Effect of Tip Gap and Squealer Geometry on Detailed Heat Transfer Measurements Over a High Pressure Turbine Rotor Blade Tip. Journal of Turbomachinery, 126, 221-228.

PFAU, A., SCHLIENGER, J., KALFAS, A. I. \& ABHARI, R. S. 2002. Virtual Four Sensor Fast Response Aerodynamic Probe (FRAP $\left.{ }^{\circledR}\right)$. The 16th Symposium on Measuring Techniques in Transonic and Supersonic Flow in Cascades and Turbomachines. Cambridge, UK.

PFAU, A., SCHLIENGER, J., KALFAS, A. I. \& ABHARI, R. S. 2003. Unsteady, 3-Dimensional Flow Measurement Using a Miniature Virtual 4 Sensor Fast Response Aerodynamic Probe (FRAP). 307-315.

REZASOLTANI, M., LU, K., SCHOBEIRI, M. T. \& HAN, J.C. 2015. A Combined Experimental and Numerical Study of the Turbine Blade Tip Film Cooling Effectiveness Under Rotation Condition. Journal of Turbomachinery, 137, 051009051009-12. 
SAXENA, V., NASIR, H. \& EKKAD, S. V. 2004. Effect of Blade Tip Geometry on Tip Flow and Heat Transfer for a Blade in a Low-Speed Cascade. Journal of Turbomachinery, 126, 130-138.

SCHÄDLER, R., HÄNNI, D. D., KALFAS, A. I., ABHARI, R. S., SCHMID, G., LUTUM, E. \& SCHNEIDER, C. Noise Characteristics of a Reduced Blade Count Rotor with Improved Stage Efficiency. GPPS Zurich, 15th-16th January 2019 Zurich.

SELL, M., SCHLIENGER, J., PFAU, A., TREIBER, M. \& ABHARI, R. S. The 2-Stage Axial Turbine Test Facility "LISA". Turbo Expo: Power for Land, Sea, and Air, 2001 New Orleans, Louisiana, USA. Volume 1: Aircraft Engine; Marine; Turbomachinery; Microturbines and Small Turbomachinery, V001T03A076.

ZHOU, C. \& ZHONG, F. 2017. A Novel Suction-Side Winglet Design Philosophy for High-Pressure Turbine Rotor Tips. Journal of Turbomachinery, 139, 111002-111002-11. 